\title{
Combined Effect of Group Based Exercise Versus Balance Step Training Among Geriatrics
}

\author{
Karthikeyan Thangavelu ${ }^{1}$, Bhadri Narayan ${ }^{2}$, Dwaraknarth Srinivas ${ }^{3}$ \\ ${ }^{1}$ Physiotherapy Unit, National Institute of Mental Health and Neurosciences, Bangalore, India \\ ${ }^{2}$ Department of Neuro Anesthesia, National Institute of Mental Health and Neurosciences, Bangalore, India \\ ${ }^{3}$ Department of Neuro Surgery, National Institute of Mental Health and Neurosciences, Bangalore, India
}

Email address:

karthik_77in@yahoo.co.in (K. Thangavelu)

To cite this article:

Karthikeyan Thangavelu, Bhadri Narayan, Dwaraknath Srinivas. Combined Effect of Group Based Exercise versus Balance Step Training Among Geriatrics. International Journal of Neurologic Physical Therapy. Vol. 4, No. 1, 2018, pp. 16-23. doi: 10.11648/j.ijnpt.20180401.13

Received: December 17, 2017; Accepted: January 4, 2018; Published: January 29, 2018

\begin{abstract}
In non- active sports geriatric population the falls deficit, which are very common, led to significant morbidity. Therapeutic intervention includes balance training or resistive exercises have been shown to improve clinical testing of balance in non- active sports geriatric population. The particular selected geriatric specific age group those who have mobility disorders or functional impairment which is used to be considered. The aim of the study to identify superior effects between group based exercise (GBE) and combined balance step training (CBST) on a therapeutic balance among non- active sports geriatric population. The methodology which is used in the study purposive sampling technique includes sixty subjects those who have more than five years both genders were included. The participants divided into two groups, Group A and Group B. Group A administered combined balance and step training while group B undergone group based exercise program. The pre and post test values of balance were assessed following the interventions. The collective data was analyzed performed by paired and unpaired ' $t$ ' test. The results of the study show that there was a significant difference existing in group A and group $\mathrm{B}$ in balance deficit. But in group A which received combined balance and step training, there was a highly significant difference as compared to group B. The present study, which revealed that combined balance and step training has a much higher significant effect on balance geriatric individuals as compared to group based exercise program.
\end{abstract}

Keywords: CBST, GBE, TUG, TB and NASGP

\section{Introduction}

One of the leading geriatric domestic cares for people over the age of sixty is falling, which is often related to balance disorder/deficit. Every year the estimated amount, between twenty and forty percent of non active sports persons over 65 who live at home, they prone to fall. The consequences of falls can be disastrous; between 12 and 67 percent of non active sports population who fracture a hip die within one year. As a result, major scientific efforts are devoted to determining the causes of falling in older adults in an attempt to reduce this significant health hazard. Balance in walking and standing is dependent on many factors. Good balance requires reliable sensory input from the individual's vision, vestibular system (the balance system of the inner ear), and proprioceptors (sensors of position and movement in the feet and legs [1]. They are prone to a variety of diseases that affect these systems, including cataracts, glaucoma, diabetic retinopathy, and macular degeneration, which all affect vision; diabetic peripheral neuropathy, which affects position sense in the feet and legs; and degeneration of the vestibular system. Balance is also dependent on good muscle strength and joint mobility. A sedentary lifestyle and arthritis or diseases of bones and muscles can compromise strength and mobility. Because balance is a complex function, there is often no single identifiable cause of falls in a geriatric population. However, geriatric population with chronic dizziness or imbalance is two to three times more likely to fall in comparison with geriatric population who do not experience these problems. Symptoms of a sense of lightheadedness or disorientation (dizziness) and/or a mild to violent spinning sensation (vertigo) can have a variety of causes: vestibular (inner ear) disorders, central nervous 
system disorders (such as stroke), cardiac problems (including low or high blood pressure), low blood sugar, infection, hyperventilation associated with anxiety attacks, medication side effects or interactions between drugs, or an inadequate or poorly balanced diet. A thorough evaluation by a physician is usually necessary to help sort out these different possible causes and arrive at a correct diagnosis. This task can be even more complicated when multiple problems are present $[1,2]$. In such cases, the trouble in any one system may not be severe, but the combined effects may be enough to cause a serious problem with balance. For example, a geriatric individual with arthritis in the ankle joints and a mild degeneration in vestibular function may be able to balance adequately until under-going an operation to remove cataracts. The disturbance in vision during the healing process and the adjustment to the new glasses or contacts may then be sufficient to result in imbalance and falls. Falls are common in the geriatric population and lead to significant morbidity. [1] An exercise program combined balance and stepping training was designed to focus on improving dynamic balance responses, increasing step length and speed, and responding safely to postural challenging situations $[1,7,8]$.

Balance exercise programs can be effective in improving gait and balance, as well as reducing falls and fall-related injuries [2, 7]. Impaired gait and balance is one of the most significant causes and consequences of falls. Exercise programs that include balance training or resistive exercises have been shown to improve clinical testing of balance in geriatric population selected for mobility disorders or functional impairment.

The primary sequelae of falls includes fall related injuries, such as fractures and head injuries, and post-fall anxiety [5]. These lead to loss of independence through the disability and fear of falling [6,8]. The reduction in mobility and independence are often serious enough to result in admission to hospital or a nursing home or even premature deaths. The role of exercise as a means of reducing falls has been the focus of considerable recent research. Supervised general group exercise also been found to be effective in moderating falls risk factors [4, 8]. Community-based group exercise programs are easily accessible, affordable and held at times and frequencies suitable for geriatric population.

\subsection{Need of the Study}

Studies have shown that group based exercise program as well as an individualized exercise program, improves balance in geriatric population with balance impairment. The group based program include some number of individuals performing exercises together, which will inculcate a feeling of competition and individuals will try to perform in a better way. The individualized program will give personal attention to every individual as it is performing the exercises and hence minute flaws if any can be corrected at the same time. Hence this study had given us the basis to know whether a group based exercise program or an individualized exercise program is better when compared for geriatric population with balance impairment.

\subsection{Objectives of the Study}

1) To evaluate the influence of combined balance and step training in geriatric population with balance impairment.

2) To evaluate the influence of the group based exercise program on geriatric population with balance impairment.

3) To identify which is more placebo effect of combined balance and step training versus group based exercise program to improve balance in the geriatric population.

\section{Methods}

\subsection{Materials Used}

1. Chalk

2. 1 meter measuring Tape

3. An arm Chair

4. Weighing Scale

5. Stop watch

\subsection{Study Design}

The study performed was a comparative study that of Quasi Experimental

\subsection{Sample Design}

The sampling design used for the study was purposive random sampling.

\subsection{Sample Size}

Sixty subjects fulfilling the inclusion and exclusion criteria were taken into the study and assigned into two groups; Group A, and Group B with 30 subjects in each group.

\subsection{Source of Data}

Sixty male and female individuals in the age group of 65 and above from various old aged homes in and around Mangalore.

\subsection{Duration of the Study}

The study was conducted over duration of 12 months.

\subsection{Inclusion Criteria}

1. Both genders with age with above 65 among nonactive sports geriatric population.

2. To be able to stand and take one step, unsupported by a device or person.

3. Unable to stand unipedally for more than 25 seconds or had at least one error in a 10- step tandem walk.

4. Body mass index pertaining to within the normal limits.

\subsection{Exclusion Criteria}

1. Fractures of the hip and knee.

2. Dislocations of the lower limb. 
3. Hamstring injuries.

4. Hyper mobility of the lower limb joint.

5. Muscle imbalances of the lower limb.

6. Nerve lesions of the lower limb.

7. Subjects have low back pain in the last 2 months.

8. Metal pins, plates, or screws in the femur.

9. Neurological abnormalities.

10. Any pathologies of lower back, hip, thigh and knee.

\subsection{Outcome Measures}

1. Berg Balance Scale,

2. Timed up and go test.

Sixty individuals among the geriatric population considered therapeutic balance training. The participants of both genders were recruited those who met fulfilling the eligibility criteria were selected. The recruited participants randomly divided into two groups i.e. group A and group B, each group consisting of 30 subjects. Informed consent approval obtained from them. Pre test was conducted on group A, and group B through Berg Balance scale and time up and go test. After a brief demonstration, Group A subjects received combined balance and step training while Group B subjects received group based exercise program.

\subsubsection{Berg Balance Scale}

The Berg Balance Scale (or BBS) is a widely used clinical test of a person's static and dynamic balance abilities, named after Katherine Berg, one of the developers. For functional balance tests, the BBS is generally considered to be the gold standard. The test takes 15-20 minutes and comprises a set of 14 simple balance related tasks, ranging from standing up from a sitting position, to standing on one foot. The degree of success in achieving each task is given a score of zero (unable) to four (independent), and the final measure is the sum of all of the scores.

\subsubsection{Timed up and Go Test}

The Timed Up and Go test (TUG) is a simple test used to assess a person's mobility and requires both static and dynamic balance. It uses the time that a person takes to rise from a chair, walk three meters, turn around, walk back to the chair, and sit down. During the test, the person is expected to wear their regular footwear and use any mobility aids that they would normally require. [2] The TUG is used frequently in the elderly population, as it is easy to administer and can generally be completed by most older adults. One source suggests that scores of ten seconds or less indicate normal mobility, $11-20$ seconds are within normal limits for frail geriatric population and disabled patients, and greater than 20 seconds means the person needs assistance outside and indicates further examination and intervention. A score of fourteen seconds or more suggests that the person may be prone to falls.

\subsection{Treatment Procedure}

\subsubsection{Combined Balance and Step Training}

Using a motor-skill training approach, thirty thirty CBST participants worked on dynamic balance and stepping responses through structured practice with a focus on speed of step initiation and appropriate toe clearance and step length. A circuit of progressive challenges to upright static and dynamic balance were presented by narrowing the base of support (bipedal to tandem to unipedal), moving from symmetrical to asymmetrical base of support, providing external challenges to balance that required a response (e.g., moving the upper extremities and bouncing and catching a ball), narrowing the base of support while responding to the external challenge (e.g., standing in semi tandem while catching a ball), and increasing the complexity of ambulatory tasks (e.g., changing direction walking backward or laterally), changing speed, walking with reduced base of support, turning, bending, stepping on and off curbs, braiding/grapevine steps, walking while carrying a ball, or stepping over obstacles (e.g., small hurdles). Functional ankle, knee, and hip strengthening activities (such as heel and toe rises and rising from a chair) was included. The use of a relatively large range of these exercises will allow progressive delivery of different levels of balance and stepping challenge.

\subsubsection{Group Based Exercise Program}

All class sessions began with the warm-up exercises. The space accommodated a large set of parallel bars and multiple chairs. Warm-up exercises were performed in a seated position with the participants' chairs being set-up in a " $U$ " shape around the instructor and facilitators. The majority of the standing exercises was performed around the parallel bars (each participant had ample room to stand and move without touching each other). Chairs were positioned behind each patient to allow them to sit and rest immediately if necessary. Recruited participants were advised to exercise at their "own level" and "listen to their body". They were encouraged to sit (chair located behind them) at any time during the class to rest if necessary and advised to inform a staff member immediately if they experienced any form of distress. The curriculum consisted of critical balance elements essential for safe performance of ADLs. These elements were progressively increased in difficulty over the 8 week period with increased class time allotted to elements which are more complex and potentially harder for subjects to master. The class began with an emphasis on core stability exercises in standing and rapidly progressed through various levels of mobility and center of gravity control over the 8 weeks; ultimately ending with multi-tasking and complex movement coordination skills.

\subsubsection{Statistical Tool Adopted in This Study}

The collected data were analyzed and computed which performed by paired and unpaired' test. 


\section{Results}

Table 1. Comparison of mean age of participants between Group A and B.

\begin{tabular}{|c|c|c|c|c|c|c|c|c|}
\hline Group & $\mathbf{N}$ & Minimum & Maximum & Mean & Std. Deviation & Median & T value & P value \\
\hline Group A & 30 & 62 & 72 & 69.13 & 4.416 & 69.00 & & \\
\hline Group B & 30 & 62 & 72 & 69.13 & 4.416 & 69.00 & .000 & $1.000 \mathrm{NS}$ \\
\hline Total & 60 & 62 & 72 & 69.13 & 4.416 & 69.00 & & \\
\hline
\end{tabular}

This table shows the age comparison between the two groups. In Group A, mean age is $69.13 \pm 4.416$. In group B, mean age is $69.13 \pm 4.416$. ' $\mathrm{t}$ ' test shows that there is no significant difference between group A and group B with respect to age.

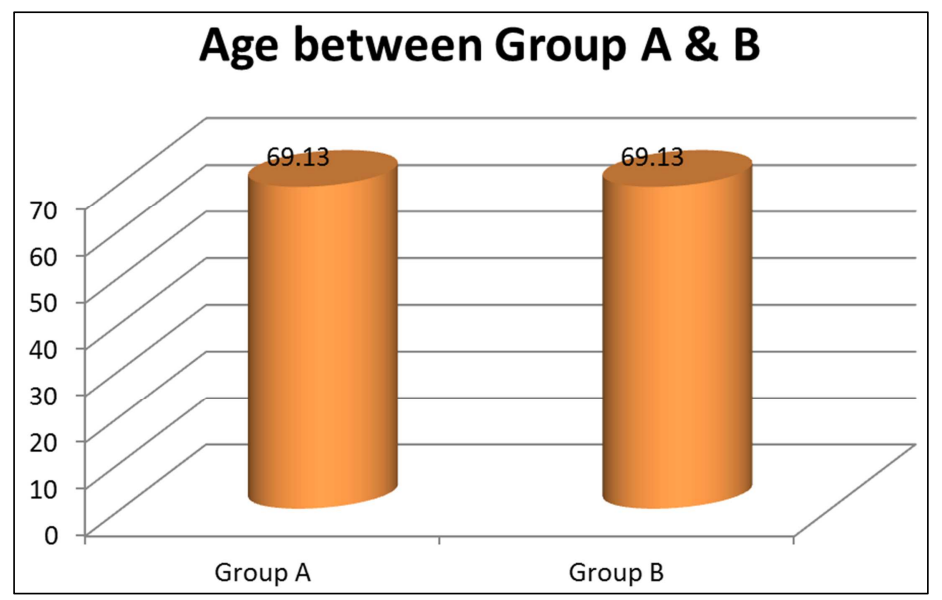

Figure 1. Comparison means age of participants between the two groups.

Table 2. Comparison of Height of the participants between the groups.

\begin{tabular}{|c|c|c|c|c|c|c|c|c|}
\hline Group & $\mathbf{N}$ & Minimum & Maximum & Mean & Std. Deviation & Median & t value & p value \\
\hline Group A & 30 & 58 & 87 & 73.83 & 7.325 & 73.00 & \multirow{3}{*}{1.211} & \multirow{3}{*}{$1.231 \mathrm{NS}$} \\
\hline Group B & 30 & 58 & 82 & 71.50 & 7.597 & 71.00 & & \\
\hline Total & 60 & 58 & 87 & 72.67 & 7.492 & 73.00 & & \\
\hline
\end{tabular}

In Group $\mathrm{A}$, mean height is $73.83 \pm 7.325$. In group $\mathrm{B}$, mean height is $71.50 \pm 7.597$. ' $\mathrm{t}$ ' test shows that there is no significant difference between group A and group B with respect to height.

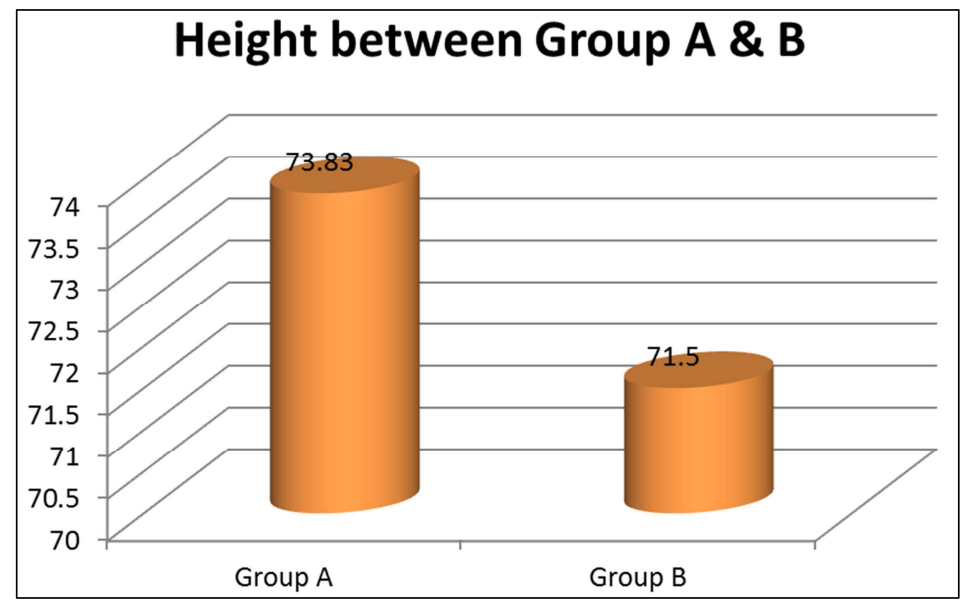

Figure 2. The comparison of Height of the participants between the two groups.

Table 3. Comparision of weight of the participants between the groups.

\begin{tabular}{lllllllll}
\hline Group & N & Minimum & Maximum & Mean & Std. Deviation & Median & T value & P value \\
\hline Group A & 30 & 56 & 87 & 66.70 & 8.069 & 66.00 & & \\
Group B & 30 & 56 & 87 & 66.70 & 8.069 & 66.00 & 0.000 & 1.000 NS \\
Total & 60 & 56 & 87 & 66.70 & 8.001 & 66.00 & \\
\hline
\end{tabular}


In Group $A$, mean weight is $66.70 \pm 8.069$. In group $B$, mean height is $66.70 \pm 8.069$. ' $\mathrm{t}$ ' test shows that there is no significant difference between group A and group B with respect to weight.

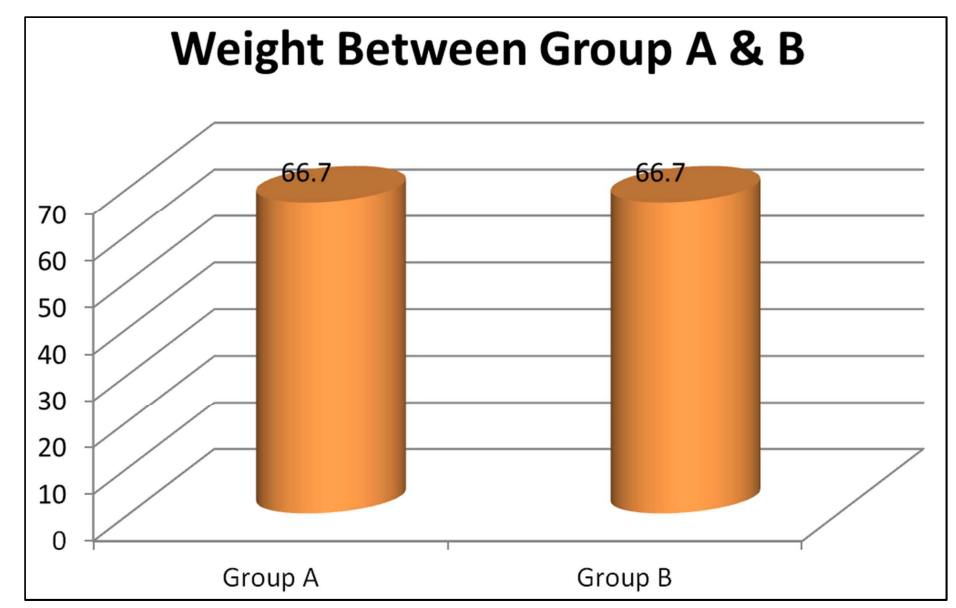

Figure 3. The comparison weight of the participants between the two groups.

Table 4. Mean and SD of BBS and TUG between Group A and B.

\begin{tabular}{|c|c|c|c|c|c|c|c|c|}
\hline Group & $\mathbf{N}$ & Minimum & Maximum & Mean & Std. Deviation & Median & T value & $P$ value \\
\hline BBS Group A & 30 & 18 & 42 & 28.67 & 6.250 & 28.00 & \multirow{3}{*}{.067} & \multirow{4}{*}{$0.946 \mathrm{NS}$} \\
\hline BBS (Group B) & 30 & 21 & 42 & 28.57 & 5.191 & 28.00 & & \\
\hline Total & 60 & 18 & 42 & 28.67 & 5.696 & 28.00 & & \\
\hline TUG (Group A) & 30 & 11 & 31 & 22.67 & 5.707 & 23.50 & \multirow{3}{*}{0.000} & \\
\hline TUG (Group B) & 30 & 11 & 31 & 22.67 & 5.707 & 23.50 & & \multirow[t]{2}{*}{$1.000 \mathrm{NS}$} \\
\hline Total & 60 & 11 & 31 & 22.67 & 5.659 & 23.50 & & \\
\hline
\end{tabular}

This table shows the comparison of pre intervention score between the groups The pre intervention values score between the groups were non significant.

Table 5. Pre to Post comparison mean score between Group A and B of BBS.

\begin{tabular}{|c|c|c|c|c|c|c|c|c|c|c|}
\hline Group & & $\mathbf{N}$ & Minimum & Maximum & Mean & Std. Deviation & Median & Change & T value & P value \\
\hline \multirow{2}{*}{ Group A } & Pre test & 30 & 18 & 42 & 28.67 & 6.250 & 28.00 & \multirow{2}{*}{37.33} & \multirow{2}{*}{12.512} & 0.000 \\
\hline & Post test & 30 & 28 & 50 & 39.37 & 6.037 & 39.00 & & & HS \\
\hline \multirow{2}{*}{ Group B } & Pre test & 30 & 21 & 40 & 28.57 & 5.191 & 28.00 & \multirow{2}{*}{7.58} & \multirow{2}{*}{6.543} & 0.000 \\
\hline & Post test & 30 & 22 & 46 & 30.73 & 5.112 & 30.00 & & & HS \\
\hline
\end{tabular}

This shows the within group-pre to post comparison in group A and group B for Berg Balance Scale. The mean pre test value in Group A was 28.67 with a SD of \pm 6.25 while the mean post test value was 39.37 with a SD of \pm 6.04 . The ' $p$ ' value was 0.000 which was highly significant. In Group B the mean pre test value was 28.57 with a SD of \pm 5.19 while the mean post test value was 30.73 with a $\mathrm{SD}$ of \pm 5.11 . The 'p' value was 0.000 which shows it was highly significant.

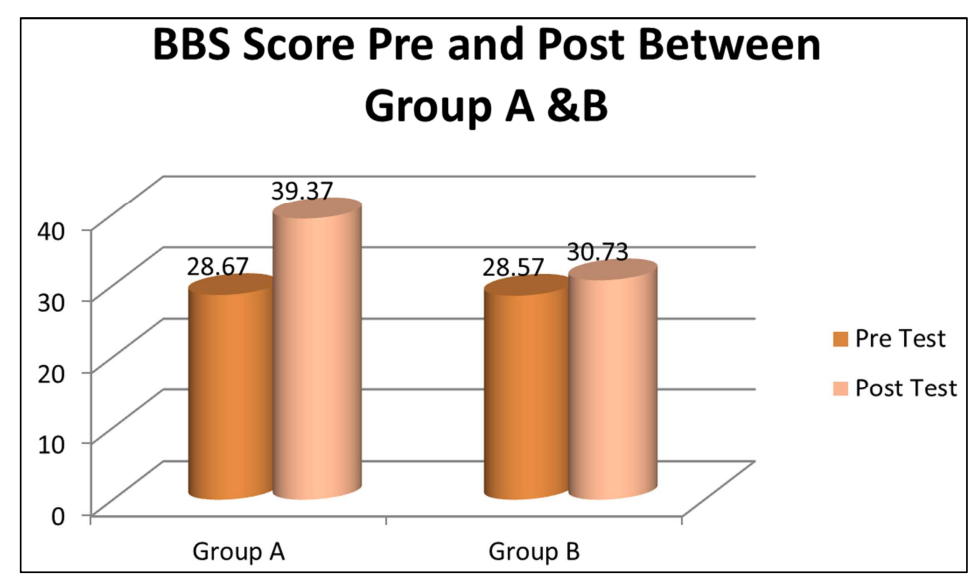

Figure 4. Pre to post comparison mean score between group A and B of BBS 
Table 6. Mean comparison between group A and B of Berg Balance Scale.

\begin{tabular}{llllllll}
\hline Group & $\mathbf{N}$ & Mean & Std. Deviation & Median & Change & Mann-Whitney Test $\mathbf{Z}$ value & p value \\
\hline Group A & 30 & 10.70 & 4.684 & 9.00 & 37.33 & \multirow{2}{*}{6.639} & 0.000 HS \\
Group B & 30 & 2.17 & 2.451 & 2.00 & 7.58 & & \\
\hline
\end{tabular}

In group A the mean of the change of effect was found to be 10.70 with a SD of 4.684 while the mean in group B was found to be 2.17 with a SD of 2.451. The 'p' value was 0.000 which shows there was a highly significant difference in the comparison of effect between the two groups in Berg Balance Scale.

Table 7. Pre to post comparison between group A and B of TUG.

\begin{tabular}{lllllllllll}
\hline Group & & N & Minimum & Maximum & Mean & Std. Deviation & Median & Change & t value & p value \\
\hline Group & Pre test & 30 & 11 & 31 & 22.67 & 5.707 & 23.50 & -22.65 & \multirow{2}{*}{4.842} & \multirow{2}{*}{$0.000 \mathrm{HS}$} \\
A & Post test & 30 & 20 & 26 & 17.53 & 4.932 & 18.00 & & & \\
Group & Pre test & 30 & 11 & 31 & 22.67 & 5.707 & 23.50 & -11.62 & 4.295 & $0.000 \mathrm{HS}$ \\
B & Post test & 30 & 11 & 30 & 20.03 & 5.288 & 20.00 & & \\
\hline
\end{tabular}

This shows the within group-pre to post comparison in group A and group B for Timed up and Go Test. The mean pre test value in Group A was 22.67 with a SD of \pm 5.70 while the mean post test value was 17.53 with a SD of \pm 4.93 . The ' $p$ ' value was 0.000 which was highly significant. In Group B the mean pre test value was 22.67 with a SD of \pm 5.709 while the mean post test value was 20.03 with a $\mathrm{SD}$ of \pm 5.28 . The 'p' value was 0.000 which shows it was also highly significant.

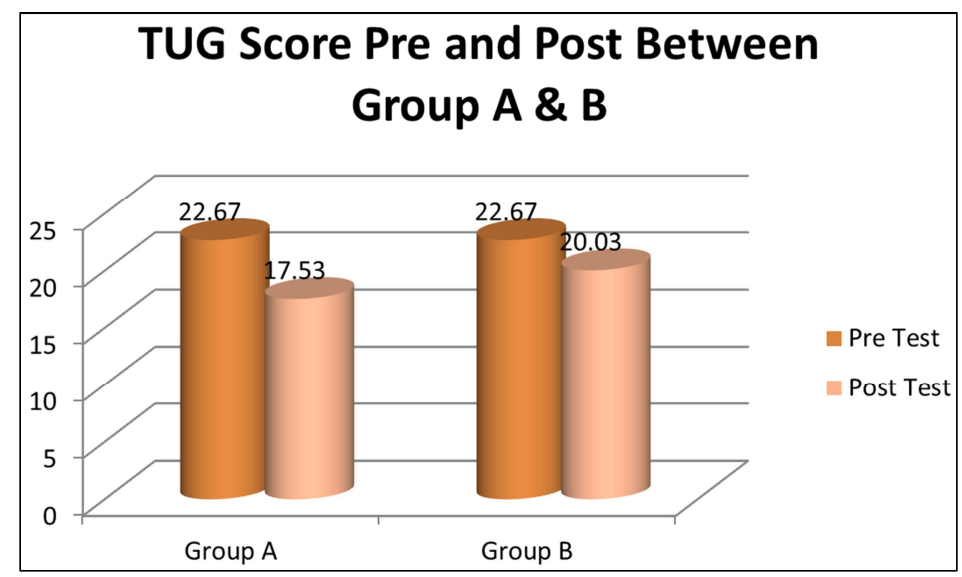

Figure 5. Pre to post comparison in group A and group B of TUG.

This diagram shows the within group-pre to post comparison in group A and group B for Timed up and Go Test.

Table 8. The comparison of effect between the group A and B of TUG.

\begin{tabular}{llllllll}
\hline Group & $\mathbf{N}$ & Mean & Std. Deviation & Median & Change & Mann-Whitney Test $\mathbf{Z}$ value & p value \\
\hline Group A & 30 & 5.13 & 4.297 & 4.00 & -22.65 & 2.849 & HS \\
Group B & 30 & 2.63 & 3.358 & 1.50 & -11.62 & 2.004 & H \\
\hline
\end{tabular}

In group A the mean of the change of effect was found to be 5.13 with a SD of 4.297 while the mean in group B was found to be 2.63 with a SD of 3.358. The 'p' value was 0.004 which shows there was a highly significant difference in the comparison of effect between the two groups for the Times up and Go test (TUG).

Table 9. The Correlation between effect of treatment and age.

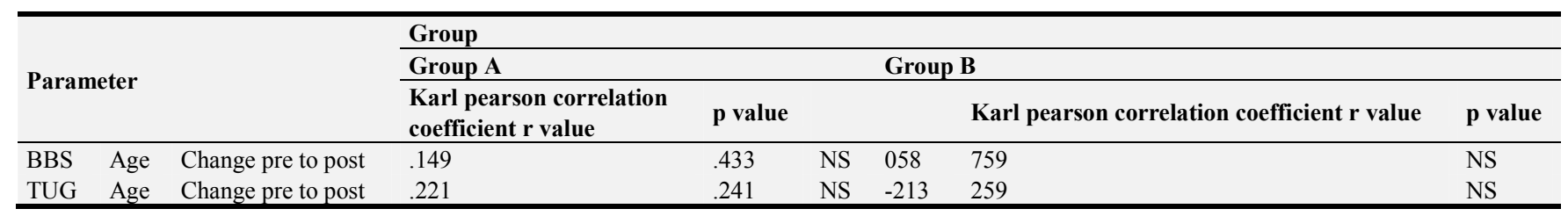

This table shows that there is no significant correlation between age and effect of treatment

\section{Discussion}

This study was designed to compare the efficacy of a group 
based exercise program and an individualized combined balance step training (CBST) program. 60 individuals above the age of 65 years, both male and females from various old aged homes in and around Mangalore were initially assessed for inclusion and exclusion criteria. Subjects were then divided into 2 groups, i.e.; Group A comprising of subjects who underwent combined balance step training and Group B subjects who underwent group based exercise program. Subjects were taken from purposive sampling. The type of study done was quasi experimental and collective data was analyzed with paired ' $t$ ' test. There was one incident where a subject was feeling nauseated while performing the get up and go test and hence was considered as a dropout.

Subjects were initially assessed for all the parameters such as height, weight, Berg balance scale and timed up and go test and the again re tested after giving the interventions to each of the group. Group A subjects received combined balance and step training while Group B subjects received group based exercise program. Using a motor-skill training approach, 30 CBST participants worked on dynamic balance and stepping responses through structured practice with a focus on speed of step initiation and appropriate toe clearance and step length. A circuit of progressive challenges to upright static and dynamic balance were presented by narrowing the base of support (bipedal to tandem to unipedal), moving from symmetrical to asymmetrical base of support, providing external challenges to balance that required a response (e.g moving the upper extremities and bouncing and catching a ball), narrowing the base of support while responding to the external challenge (e.g., standing in semi tandem while catching a ball), and increasing the complexity of ambulatory tasks (e.g., changing direction walking backward or laterally), changing speed, walking with reduced base of support, turning, bending, stepping on and off curbs, braiding/grapevine steps, walking while carrying a ball, or stepping over obstacles (e.g., small hurdles)). Functional ankle, knee, and hip strengthening activities (such as heel and toe rises and rising from a chair) was included. The use of a relatively large range of these exercises will allow progressive delivery of different levels of balance and stepping challenge. All class sessions began with the warm-up exercises. The space accommodated a large set of parallel bars and multiple chairs. Warm-up exercises were performed in a seated position with the participants' chairs being set-up in a " $U$ " shape around the instructor and facilitators. The majority of the standing exercises was performed around the parallel bars (each participant had ample room to stand and move without touching each other). Chairs were positioned behind each patient to allow them to sit and rest immediately if necessary. Patients were advised to exercise at their "own level" and "listen to their body". They were encouraged to sit (chair located behind them) at any time during the class to rest if necessary and advised to inform a staff member immediately if they experienced any form of distress. The curriculum consisted of critical balance elements essential for safe performance of ADLs. These elements were progressively increased in difficulty over the 8 week period with increased class time allotted to elements which are more complex and potentially harder for subjects to master. The class began with an emphasis on core stability exercises in standing and rapidly progressed through various levels of mobility and center of gravity control over the 8 weeks; ultimately ending with multi-tasking and complex movement coordination skills. The outcome measure was the Berg Balance scale and timed up and go test which was given to both the groups before and after the interventions. The readings from both the groups were then analyzed by ' $t$ ' test. The results showed that there is no significant difference between the two groups ie Group A and Group B with respect to age. Also there is no significant difference between Group A and B with respect to weight. Also based by the results it is clear that both combined balance based step training and group based exercise program have significant effects on the balance. However the effect of combined balance step training is significantly higher than that of the group based exercise program.

\section{Conclusion}

Combined balance step training significantly improves the balance in the geriatric population. Group Based exercise program significantly improves the balance in the geriatric population. When compared, combined balance step training produces a much higher improved performance in balance in geriatric population with respect to the group based exercise program. In conclusion, it was evident that combined balance step training produces a much higher improved performance in balance in geriatric population with respect to the group based exercise program.

\section{References}

[1] Nnodim JO, Strasburg D, Nabozny M, Nyquist L, Galecki A, Chen S, Alexander NB. Dynamic balance and stepping versus tai chi training to improve balance and stepping in at-risk older adults. Journal compilation, The American Geriatrics Society Dec; 2006, 54 (12): 1825-31.

[2] TatjanaBulat, Stephanie Hart-Hughes, Shahbaz Ahmed, Pat Quigley, Polly Palacios, Dennis C Werner, and Philip Foulis. Effect of a group-based exercise program on balancein elderly. ClinInterv Aging. December; 2007, 2 (4): 655-660. Published online 2007 December.

[3] Interventions for the prevention of falls in older adults: systematic review and meta-analysis of randomized clinical trials. BMJ 2004; 328: 680 .

[4] Lord S, Ward J, Williams P, Strudwick M. The effect of a 12month trial on balance, strength, and falls in older women: A randomized controlled trial. J Am GeriatrSoc1995; 43: 1198206.

[5] Jayne Steadman, Nora Donaldson et al. A randomized control trial of an enhanced balance training program to improve mobility and reduce falls in elderly patients. Journal of American geriatric society vol 51, issue 6. june 2003. 
[6] Teresa Liu-Ambrose, Karim M. Khan et al. Resistance and Agility Training Reduce Fall Risk in Women Aged 75 to 85 with Low Bone Mass: A 6-Month Randomized, Controlled Trial. Journal of the American Geriatrics Society. Volume 52, Issue 5, pages 657-665, May 2004.

[7] Bellew JW, Fenter PC, Chelette B, Moore R, Loreno D.
Effects of a short-term dynamic balance training program in healthy older women. J GeriatrPhysTher. 2005; 28 (1): 4-8, 27.

[8] Becker C, Lindemann U, Nikolaus T. Multifactorial intervention on falls and fractures in nursing homes [abstract]. Age and Ageing2000; 29 Suppl 2: 18. 